\title{
Scope of Breast Imaging in Developing Countries
}

\author{
Hari $S^{1}$, Srivastava $A^{2}$, Thulkar $S^{1}$ \\ ${ }^{1}$ Department of Radiodiagnosis and Imaging, ${ }^{2}$ Department of Sugery AIIMS, New Delhi, \\ India
}

\section{Introduction}

Breast imaging is an important subspecialty of radiology. Modern diagnosis, treatment and follow-up of breast cancer cannot be imagined without significant contribution of breast imaging at every step and hence, expert breast radiologists are making their presence felt.

Mammography and ultrasound are the firstline investigations for imaging the breasts. MRI is a valuable adjunctive diagnostic tool because of its high sensitivity for breast pathology. Breast interventions, predominantly image guided breast biopsies, are also an important part of breast imaging practice.

\section{Breast cancer screening}

Mammography is the standard modality for community breast cancer screening in asymptomatic women. Sensitivity of mammography in detection of breast cancers in the screening set up ranges from 83 to $95 \% .^{1}$ However, it decreases to as low as 30 48 percent in patients with radiographically dense and glandular breast. $^{2}$ Several randomized controlled trials which included

Corrospondence to: Dr. Smriti Hari, Department of Radiodiagnosis and Imaging, AIIMS, New Delhi, India.

E-mail:- smritihari@ hotmail.com hundreds of thousands of women from different populations and having follow-up of decades are available in literature. ${ }^{3,4}$ No other imaging modality other than mammography has been subjected to such a large, well designed and extensive research.

These studies have established the efficacy of mammographic screening. Although right from the inception of screening, there are controversies regarding exact quantum of benefits, optimal age to start screening, over diagnoses and cost effectiveness of mammographic screening, these are largely generated from variable and sometime premature analysis of data. ${ }^{5}$ Mammographic screening reduces deaths from breast cancer and that is why it is being widely practiced in developed countries for a long time.

Mammography screening for early detection of breast cancer is however, a resource intensive proposition. Although few developing countries have started mammographic screening studies, it is not a viable option for most of the developing countries. Limited availability of mammography units and trained manpower, large population, inadequate financial allocations and other more pressing medical needs are the usual factors which do not justify mammographic screening in developed countries. 

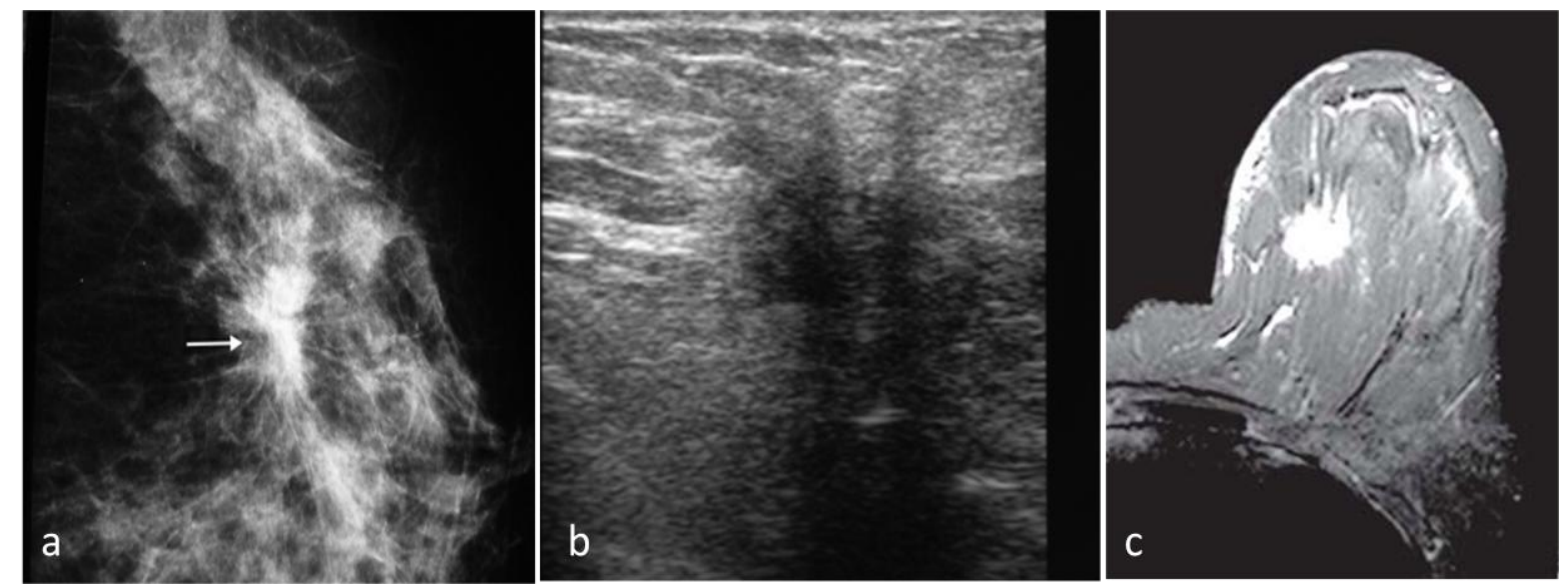

Fig. 1a, b \& c: Breast cancer on imaging: (a) Mammogram shows a high density, irregular, spiculated mass (arrow). (b) Ultrasound shows a hypoechoic, irregular, spiculated mass with distal acoustic shadowing. (c) Contrast enhanced T1 weighted MRI scan of another woman shows a spiculated, intensely enhancing mass.
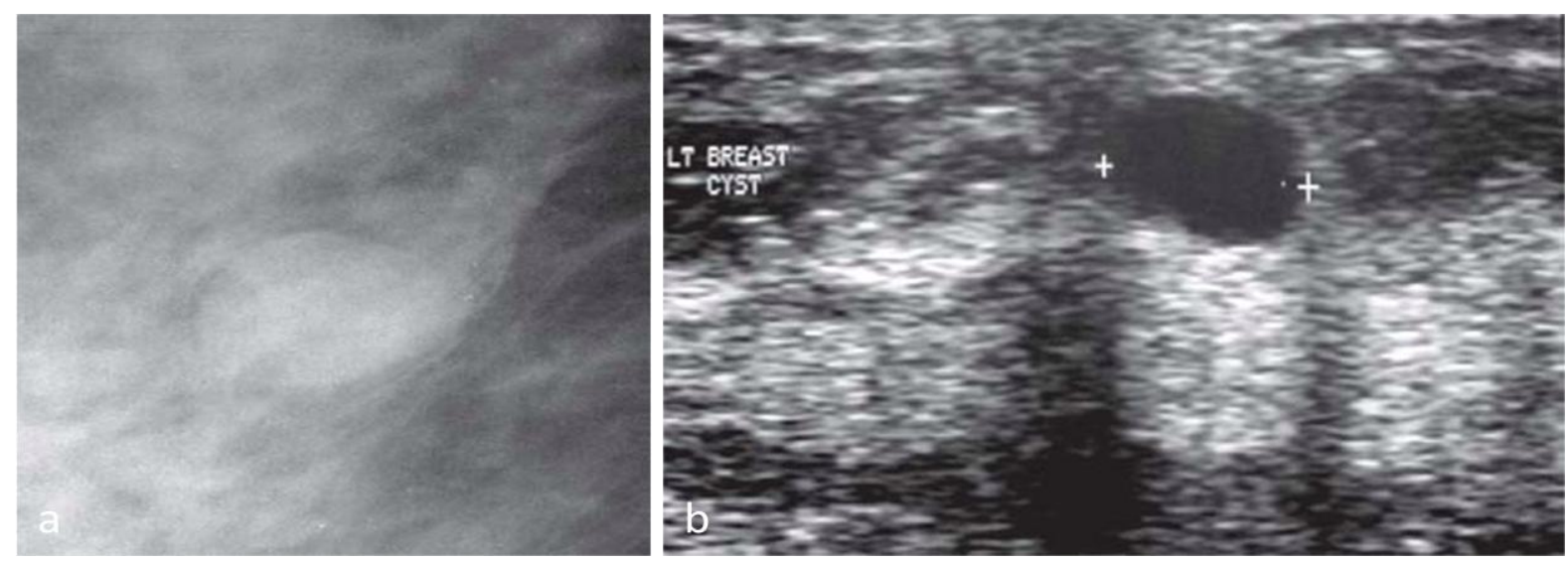

Fig. 2 a \& b: Breast cyst: (a) mammogram shows a small, round, sharply circumscribed, low density mass. (b) Ultrasound shows sharply defined, anechoic cyst with distal acoustic enhancement.
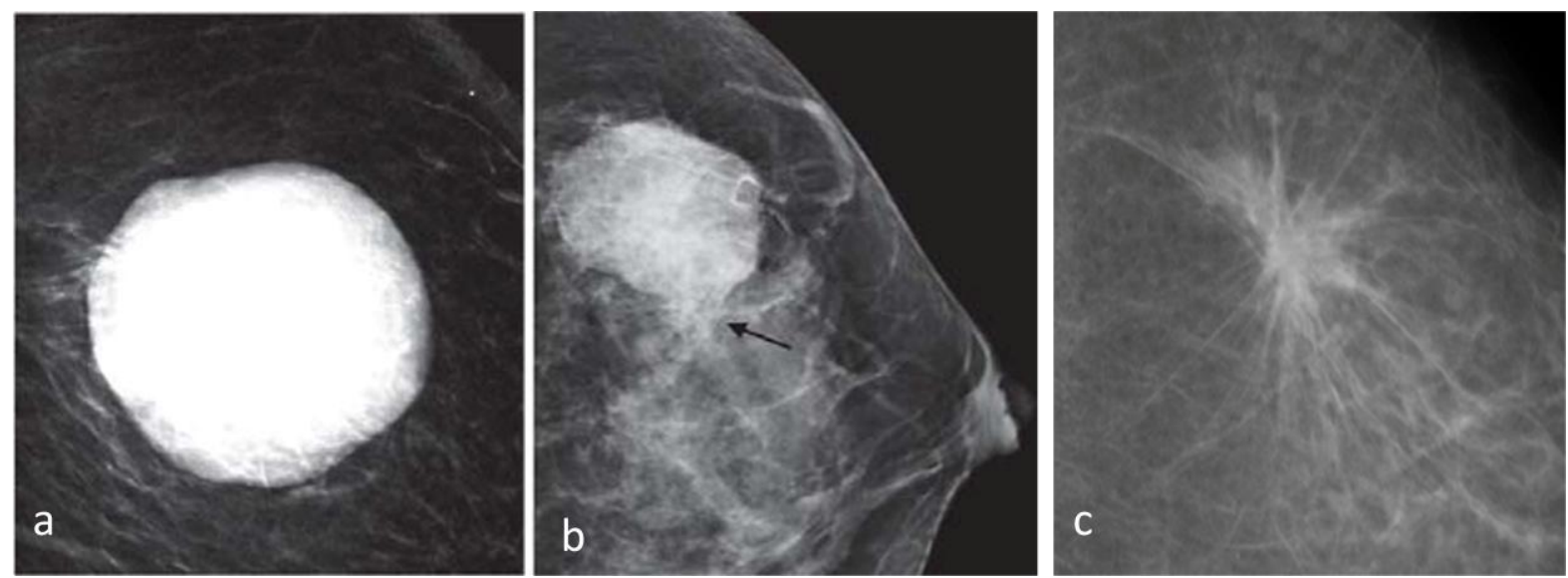

Fig. 3 a, b \& c: Margins of Breast masses on Mammography. (a) Circumscribed (sharply defined), probably benign (b) slightly Indistinct, suspicious (c) Spiculated, highly suspicious for malignancy. 


\section{Diagnostic Breast Imaging}

Non-availability of mammographic screening does not undermine the importance and need of quality breast imaging in less resourceful countries. Apart from screening, breast imaging is required to evaluate diseases of the breast in women presenting with breast related symptoms such as lump, mastalgia and nipple discharge. It is also required preoperatively in women already diagnosed with breast cancer, to define extent of the index tumor and detect additional masses as this may have implications in management decisions. Women already treated with breast cancer need regular surveillance with breast imaging to detect recurrences or metachronous breast cancers. As breast cancer is most common cancer in women, those presenting with metastatic cancer anywhere the body with occult primary needs breast imaging to confirm or exclude breast cancer as a primary site. This type of breast imaging is commonly referred to as diagnostic breast imaging and this is the major area of breast imaging in nonscreening setup.

\section{Challenges in Breast Imaging in Developing Countries}

Major challenges in developing countries include limited availability of mammography and well experienced radiologists. Symptomatic women may present to surgeons, oncologists, gynaecologists, internists or family physicians and there may be variable trends in referral for breast imaging. Utilization of breast imaging in such a setup is also governed by beliefs of the clinician and quality of breast imaging services available to them. In this situation, breast imaging does not form a major part of radiology practice and hence, there are not many full time dedicated breast radiologists. In most situations, a general radiologist with little time and experience in breast imaging has the responsibility to cater to these patients. There are also wide variations in type and quality of mammography units. Screen-film, CR based and at major centres, Full Field Digital mammography (FFDM) systems are available, in most parts of the developing countries. However, lack of mandatory Quality Assurance (QA) requirement leads to non- uniform (mostly poor) quality and this conversely affects the output and contribution of breast imaging in diagnostic setting.

A systematic approach is the key to standardize and evolve diagnostic breast imaging. Any type of mammography unit can be suitable for high quality breast imaging as long as it is be subjected to periodic QA checks. In CR or FFDM mammography systems, hard copies on film may be required for the use of patients and referring clinicians, however, reporting should be performed on mammography quality monitors, preferably 5megapixel or more, whenever available. Different formats and lexicon of reporting are commonly seen and this may decrease the confidence of clinicians on breast imaging. BI-RADS reporting system developed by ACR is a structured reporting format and lexicon developed by American College of Radiology (ACR). ${ }^{6}$ It is now widely used in most parts of the world. It is useful to achieve uniformity and minimize ambiguity in reporting.

Mammography is the primary modality of diagnostic breast imaging. Ultrasound is the 


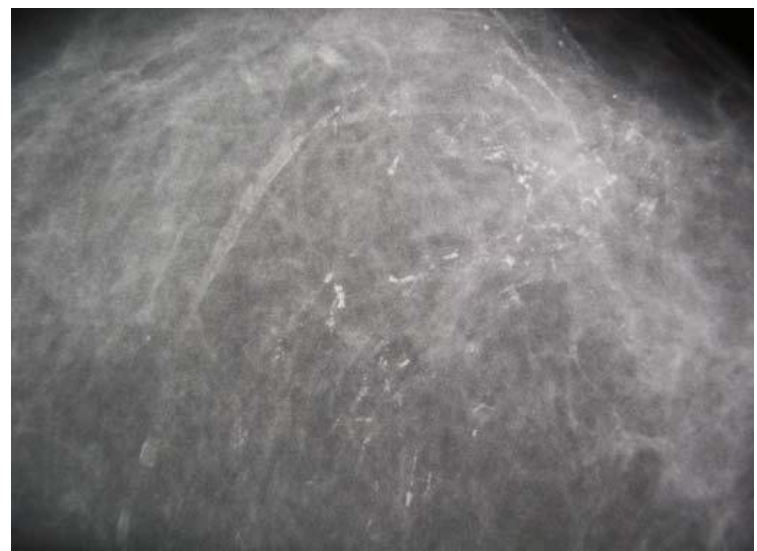

Fig. 4: Highly suspicious Calcifications on mammography; Linear branching Pleomorphic calcifications which are irregular, smaller than $0.5 \mathrm{~mm}$.

most important complimentary imaging modality to supplement mammography in diagnostic breast imaging. However, ultrasound may also be used as the first investigation in women below 30 years of age, pregnant or lactating women, in mammographically dense breasts and in those presenting with nipple discharge. Ultrasound is also the preferred modality to perform image guided breast biopsies. MRI and PET-CT are uncommonly required; however, access to these facilities is critical whenever indicated.

Although largely guided by local practices, various guidelines are available on how to optimally utilize different breast imaging modalities in diagnostic setup. These need to be followed and referring clinicians should be sensitized about indications and limitations of various imaging modalities in different clinical situations.

\section{Women Presenting With Breast Symptoms}

Breast lump felt by the patient, breast pain and nipple discharge are the common symptoms for which women seek medical assistance. Breast cancer is a major concern in adult women presenting with breast symptoms. Detailed history of their complaints and clinical breast examination is standard part of the initial evaluation and depending on clinical judgement; the patient may or may not be referred for breast imaging.

\section{Breast Lump}

Breast lump is the most common breast symptom. Most breast lumps in young women are benign such as simple cysts or fibroadenomas however; breast cancer is the most important cause in women more than 30 years of age.

Triple assessment is the standard protocol for accurate diagnosis of breast lump. It consists of CBE, imaging (ultrasound and/or mammogram) and FNAC or biopsy. A triple assessment is taken as positive if any of these is positive/suspicious and negative results on all of these are considered as a negative result for breast cancer. Triple assessment has more than $99 \%$ sensitivity in diagnosis of breast cancer with consistent reproducibility even in developing countries. $^{7,8}$

CBE is the first step in evaluation of lump and a mass is confirmed if a three dimensional structure distinct from surrounding breast parenchyma is felt. Hard, immobile and ill defined masses suggest malignancy. Associated skin and nipple changes also support the clinical diagnosis of malignancy. Discrete, smooth, soft masses are benign. However, there is significant overlap in benign and malignant masses on CBE. Moreover, there is significant 
interexaminer variation. ${ }^{9}$ Hence, CBE needs to be supplemented with imaging.

Ultrasound is the primary imaging modality in young women (less than 30 years) presenting with breast lump. Mammography is not required. If it shows a definitely benign process such as simple cyst, further work-up, biopsy or follow-up can be safely avoided (Fig. 2). If a solid mass with extremely low likelihood of malignancy such as fibroadenoma is seen in young women, reassurance and imaging follow-up would be sufficient. $^{10}$

Mammography should be performed first in older women (more than 30 years) presenting with breast lump. It is important to note that a clinically palpable breast cancer may not be demonstrated on mammogram in a minority of patients. Some of these may be apparent on ultrasound. ${ }^{11}$ Suspicious mass on any of these must be subjected to FNAC or biopsy. If the lump is demonstrated to be a simple cyst on ultrasound, it is reliable. Aspiration of the cyst resulting into complete disappearance of the lump clinically also confirms the diagnosis of simple cyst. Routine cytology of the aspirated fluid is unnecessary. A solid and palpable mass is generally assigned BIRADS IV category on imaging and accordingly, subjected to biopsy. Results of FNAC or biopsy must be correlated with $\mathrm{CBE}$ and imaging findings. A negative pathological result in a highly suspicious mass clinically or on imaging warrants rebiopsy.

\section{Mastalgia}

Breast pain or mastalgia is the second most common breast symptom. Mostly, it is bilateral and experienced by the woman in premenstrual phase in cyclical manner. It is considered to be caused by aberrant response of breast tissue to cyclical hormonal variation and is not a true disease. Indeed it may occur in one breast or at times, only in part of the breast. It may or may not be associated with feeling of heaviness or vague lump by the patient. This clinical presentation was commonly diagnosed as fibrocystic disease, but now most would like to avoid this term. Apart from local or diffuse tenderness, clinical breast examination is generally normal. Sometimes, nonspecific nodularity may be felt at site of lump complained by the patient.

Noncyclical mastalgia may be related to Oral Contraceptive use or hormone therapy, some psychotrophic and cardiovascular drugs and large pendulous breasts not supported with well fitted brassiere. ${ }^{12}$

In patients presenting with mastalgia and no mass on clinical examination, breast imaging is mainly required for excluding the existence of true underlying lesion and reassuring the patient. ${ }^{13}$ Ultrasound is the modality of choice in young women to exclude significantmass or any other pathology. Cysts or hypertrophied periductal glandular tissues may be seen on ultrasound, however, there is significant overlap of this finding with normal women and hence, this finding is of no diagnostic use. Mammography is recommended in women more than 30 years of age.

Mastalgia not associated with clinical and imaging abnormality needs reassurance as most of the times it will resolve spontaneously. Others may respond to non steroidal anti inflammatory drugs. Refractory 
cases are treated with Danazol ${ }^{13}$ Centchroman, a weak antiestrogen drug, is also effective. ${ }^{14}$

\section{Mastitis}

Mastalgia, with or without lump, may also be caused by mastitis. Breast abscesses are commonly seen in lactating women. In others, duct ectasia is an important cause of breast abscess. Mammography is difficult to perform in women with tender breasts. Ultrasound is a preferred modality for imaging evaluation and localization of breast abscess. Symptomatology and clinical findings are sufficient to make the diagnosis of acute mastitis and appropriate treatment is started. Chronic mastitis without well formed abscess is seen in idiopathic granulomatous mastitis or tubercular mastitis. Interestingly, both of these conditions are more common in developing countries than in the western world. ${ }^{15}$ Idiopathic granulomatous mastitis is generally seen in young women in their reproductive age who present with firm breast lump, often with inflammation of overlying skin. Sinus formation, invasion of chest wall and axillary lymphadenopathy may also be seen. Presentation of tubercular mastitis is also similar and both of these can mimic malignancy on $\mathrm{CBE}$ as well as imaging. Mammography shows variable findings ranging from ill defined mass, asymmetric density or diffuse breast edema. Ultrasound is useful to localize and characterise the abnormality. It shows hypoechoic confluent, ill-defined heterogeneous collections with tubular extensions and enlarged axillary nodes. These finding are non-specific and cannot be differentiated from malignancy. These conditions are rarely diagnosed on FNAC and hence, image guided core biopsy is required to rule out malignancy and arrive at the definitive diagnosis.

Inflammatory breast cancer (IBC) is the aggressive form of invasive duct carcinoma and accounts for $2.5 \%$ all breast cancers. Histopathologically, there is diffuse infiltration and obstruction of dermal lymphatics. It is characterized by younger age, rapid progression, early metastases and high mortality. Patients present with enlarged painful breast with diffuse erythema, edema, sloughing and ulcerations of the skin over the breast. Clinical presentation mimics acute mastitis and the tumour though sizable, is difficult to palpate.

The diagnosis is usually clinical. Mammography is difficult to perform in a painful breast. Usual mammographic finding is of a dense edematous breast with skin and trabecular thickening. Hence, primary tumour is uncommonly identified on mammography. Ultrasound is useful and it locates the primary tumour in more than half of patients. It is seen as irregular or lobulated mass on ultrasound, usually large in size. Multicentric and diffusely infiltrating IBC is not localized on ultrasound. MRI and PETCT are most accurate for evaluation of primary tumour as well as distant metastases. MRI detects primary tumour in nearly all patients with IBC. The primary tumour is most often seen as single or multiple confluent masses with wash out kinetics and skin involvement in majority of lesions.

\section{Nipple Discharge}

Nipple discharge or visible abnormalities of nipple-areola complex are another important group of women with breast symptoms. Nipple discharge is considered pathological 
when it is unilateral, single duct or bloody. Duct papilloma, duct ectasia and breast cancer are important causes of unilateral duct discharge. Bilateral spontaneous serous nipple discharge usually suggests systemic causes such as prolactinoma or certain drugs. Pathological duct discharge should be subjected to cytological examination to look for atypical cells. Clinical utility of fluid cytology is limited as negative result is of no value. ${ }^{16}$ Surgical duct excision is the gold standard for diagnosis of cause of duct discharge and also for the treatment if the cause is benign.

As cancer is an important concern, mammography is the initial modality for investigation. Ductal carcinoma in situ (DCIS) with or without invasive component is the commonly associated malignant diagnosis with duct discharge. Mammography has high sensitivity for its detection when it is seen as pleomorphic micro calcifications in a linear or segmental distribution. Papillomas may also be identified as suspicious masses. Ductography was the standard investigation for evaluation of single duct discharge; however, it is fast being replaced with high resolution ultrasound and MRI. ${ }^{17}$ Ultrasound is accurate in delineating the abnormally dilated duct often with an intraductal nodule or mass. If identified, its size, location on clock face and distance from the nipple should be carefully documented in the report to help the surgeon in planning the extent of duct excision (microdochectomy instead of radical duct excision). MRI is rarely required and generally advised if suspicion for cancer is high and mammography and ultrasound are negative. MRI improves the cancer detection rate in women with pathological nipple discharge.
Although, most women with pathological duct discharge require surgical duct exploration, a prior careful imaging evaluation is necessary. If benign or malignant cause is identified on imaging, surgical decision is modified accordingly.

Patients with normal clinical and imaging finding who do not desire duct excision can be subjected to short term follow-up. ${ }^{18}$

Paget's disease is an important cause of nipple discharge. It is a centrally located ductal carcinoma growing along the ducts into the nipple with eczematous changesat the summit of the nipple.Fifty per cent patientshave a palpable mass. On mammography and ultrasound, underlying mass or calcification is seen in most patients. MRI is required to localize the mass and define its extent. ${ }^{19}$ Benign calcifications may sometimes be seen in nipple areolar complex and these are located in skin, hair follicle or Montgomery glands.

It is important to note that nipple areolar complex and retroareolar area is a challenging area for mammographic evaluation. $\mathrm{CBE}$ is more sensitive for detection of cancers in this region. ${ }^{20}$

\section{Occult Primary Breast Cancer}

Mammography is standard part of work up in women presenting with metastatic adenocarcinoma anywhere from an occult primary site. Women presenting with axillary lymphadenopathy only are also evaluated with mammography. If clinical examination and mammography are negative but axillary lymph node biopsy is suspicious of primary breast carcinoma, MRI is recommended. MRI identifies mammographically occult breast cancer in 
majority of these patients and allows their treatment with $\mathrm{BCT}^{21}$ If primary is not identified, the patients are treated with mastectomy or radiotherapy. Although metastatic breast cancer is a stage 4 disease, excision of the primary offers survival advantage.

\section{Imaging features of breast cancer}

Breast cancer can manifest on mammography in several ways; mass, calcifications, architectural distortion, asymmetry or any combination of these. Ancillary signs of malignancy like lymphadenopathy, breast edema, skin or areolar thickening or retraction may be seen in advanced cases. The mammographic abnormalities are not specific for cancer and based on the morphology, these may elicit various degrees of suspicion for breast cancer. Biopsy is mandatory for definitive diagnosis of the breast cancer.

Mass is a space occupying lesion in the breast which is seen in two views. Lesion seen in only one view is considered a density unless it's three dimensional existences is established. Masses are assessed for their size, shape, margins and density. The shape may round, oval, lobulated or irregular. Margins are generally described as circumscribed (sharply defined), indistinct, spiculated or microlobulated, the later three usually, but not always, are malignant masses (Fig. 3). When all or part of the margin is abutting the normal glandular parenchyma and not amenable to precise evaluation, the term obscured margin is used. The density is described as high, equal or low relative to glandular breast parenchyma. Density of the mass is not very helpful for characterization of the masses. Exception is fat containing masses which are always benign.

Calcifications are commonly encountered on mammograms and majority of these are benign. Calcifications are evaluated for their number, size, shapes, distribution and any characteristic morphology. Calcifications are broadly classified into typically benign, intermediate concern and high probability of malignancy. Most benign calcifications are larger than $0.5 \mathrm{~mm}$. Typically benign calcifications include skin calcifications, vascular calcifications, popcorn calcifications of involuted fibroadenoma, eggshell or rim calcifications, Long rod like ductal calcifications, coarse dystrophic calcifications. Solitary or clustered (five or more in one cc of breast tissue) micro calcifications are also benign if these are round and uniform in morphology. Breast cancers do not have fatty component and hence, similar to masses, any calcification with central lucency is always benign. Amorphous or indistinct calcifications are of intermediated concern. Calcifications with high probability of malignancy are smaller than $0.5 \mathrm{~mm}$ and are pleomorphic (varying sizes and shapes), fine linear (Fig. 4) and branching casts and those which are more conspicuous than amorphous calcifications. Distribution also modifies the characterization of the calcifications which are not typically benign. Calcifications which are distributed over large area of the breast (regional) or diffusely scattered over entire or both breasts are benign, while those with segmental distribution confirming to a duct territory, those with linear branching pattern suggesting intraductal spreadand clustered fine irregular calcifications are suspicious for malignancy. 
The term architectural distortion is used to describe focal distortion or tethering of the septae, focal spiculations or the retraction of the parenchyma, in absence of a mass. Focal asymmetry is a term used to describe the asymmetric glandular density parenchyma which is appreciated when compared with same area of the contralateral breast. It usually represent an asymmetrical but normal breast tissue, however it must be ensured that it does not represent a mass. The focal asymmetry is suspicious if it is associated with palpable abnormality or on serial mammograms; it is a new or evolving finding. ${ }^{22}$

\section{Mammography reporting}

In order to achieve uniformity and objectivity in the interpretation and reporting of the mammograms, American College of Radiology has developed standard guidelines which are increasingly being used. It is called BI-RADS (Beast Imaging Reporting and Data System). ${ }^{23}$ The mammographic assessments are categorized from BI-RADS 0 to BI-RADS 6 , which are primarily aimed at communicating significance of the mammographic findings to the referring physician and to recommend the most appropriate management. BI-RADS category 0 represents incomplete evaluation when repeat mammography, additional views or investigations like ultrasound or MRI (but not biopsy) is recommended. Category 1 is normal mammogram and category 2 is definitely benign finding such as benign calcification or a fat containing mass. No further management is required. Category 3 is a probably benign finding with extremely low chances of malignancy. Short term follow up is recommended in these. Category 4 represents findings with low to high concern for malignancy and category 5 is reserved for characteristic mammographic findings with very high probability of malignancy. Biopsy is recommended for both categories 4 and 5 .

BI-RADS 6 category is used for biopsy proved malignancy when breast imaging is being performed as part of pre-treatment work-up. BI-RADS also incorporates breast imaging lexicon i.e. standard terms and nomenclature for description, analysis and interpretation of breast lesions. Originally developed for mammography only, it is now also available for ultrasound and MRI examinations of the breast. BI-RADS is still evolving and many consider it as an optimal structured reporting format, which may serve as model for other imaging modalities. Apart from standardization, such reporting formats also help to minimize the ambiguity from the reports and make the results reproducible and comparable.

\section{Breast Interventions}

Breast imaging is also required to guide biopsies from non-palpable lesions, deployment of clips before starting neoadjuvant chemotherapy and for preoperative wire localisation of nonpalpable lesions.

\section{Image guided Biopsy}

Whether detected clinically or on imaging, all suspicious breast lesions (BI-RADS 4 and 5 Categories) must be subjected to breast biopsy; Fine Needle Aspiration Cytology (FNAC) or Core Needle Biopsy (CNB).

FNAC is a simple, less expensive and less traumatic technique. However, FNAC has 

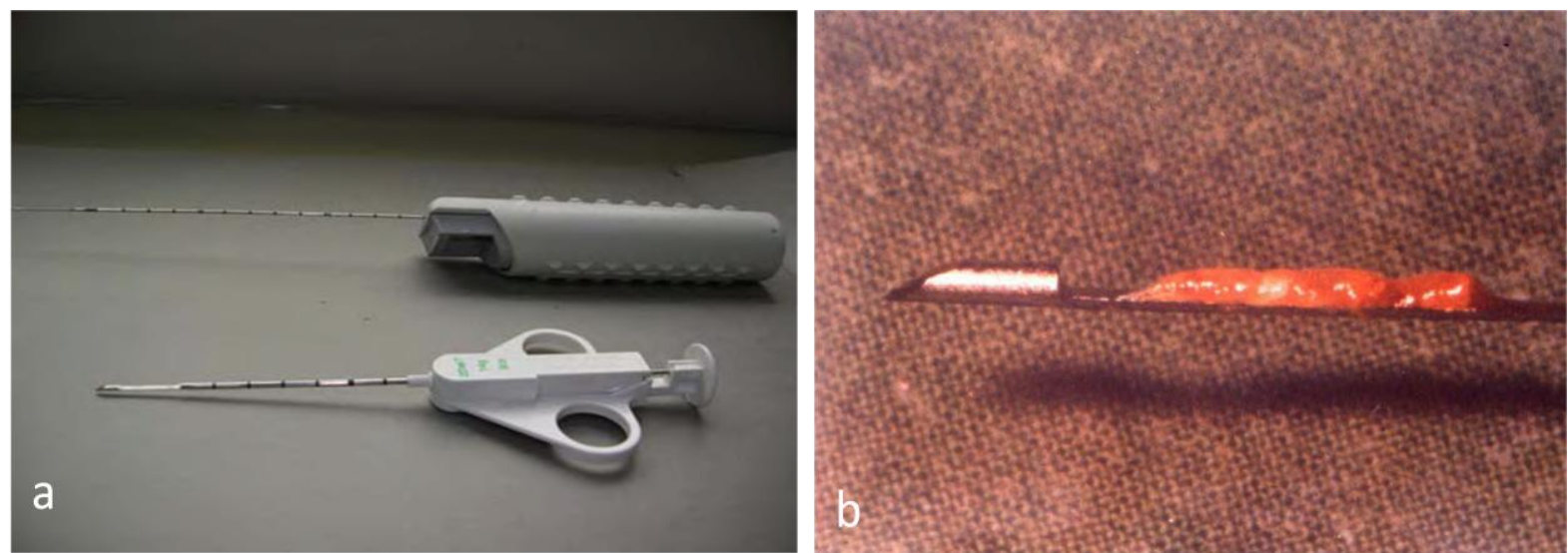

Fig. 5a \& b: Core needle biopsy of breast: (a) 14G biopsy guns (b) open sample notch of the biopsy gun showing harvested tissue specimen.

several limitations. It requires the availability of an experienced breast cytologist who can establish the adequacy of aspirated sample on site and also interpret it accurately. It has high insufficiency and false negative rates. A negative FNAC cannot reliably exclude the presence of breast cancer. FNAC is especially unsuitable for non palpable breast lesions as up to $34 \%$ of FNAC samples may be insufficient for diagnosis. ${ }^{24}$ FNAC gives cytological diagnosis only i.e. it cannot differentiate in- situ from invasive cancer. Modern treatment protocols often require immuno-histochemical analysis for hormonal receptor status and this is difficult to obtain on FNAC. Benign FNAC results are often nonspecific and definitive diagnosis of a benign condition is rarely made. This affects the radiologic-pathologic correlation and may be a limitation in deciding further management. Diagnosis of papillary lesions is inaccurate on FNAC and it cannot differentiate fibroadenoma from phyllodes tumour.

Several of the disadvantages of FNAC have been addressed by CNB of the breast and this has become the standard of care for biopsy of breast lesions; whether palpable or not. Accuracy of CNB is better than FNAC and it allows cellular and architectural evaluation. With this, specific diagnosis of benign and malignant conditions can be made. The cores are also suitable for immuno-histochemical and genetic analysis. However, CNB of breast is more traumatic, more expensive and requires higher skills to perform when compared with FNAC. Results of CNB are not immediately available and take few days at least.

Most breast biopsies are performed under imaging guidance. Mammography (stereotactic) and ultrasound are the most commonly used guiding modalities (Fig. $5,6)$. If the lesion is well demonstrated on ultrasound, it is preferred over stereotaxy, although selection of guiding modality for breast biopsy is generally a matter of operator preference and expertise. Most lesions are suitable for percutaneous biopsies. Previously very small lesions $(<5$ $\mathrm{mm}$ ) were not subjected to core biopsy for the fear of completely removing the lesion during biopsy. If the results are positive then the exact location of the tumor will not be determined and hence therapeutic excision of the tumor site becomes difficult. Now metallic clip markers are available which can 

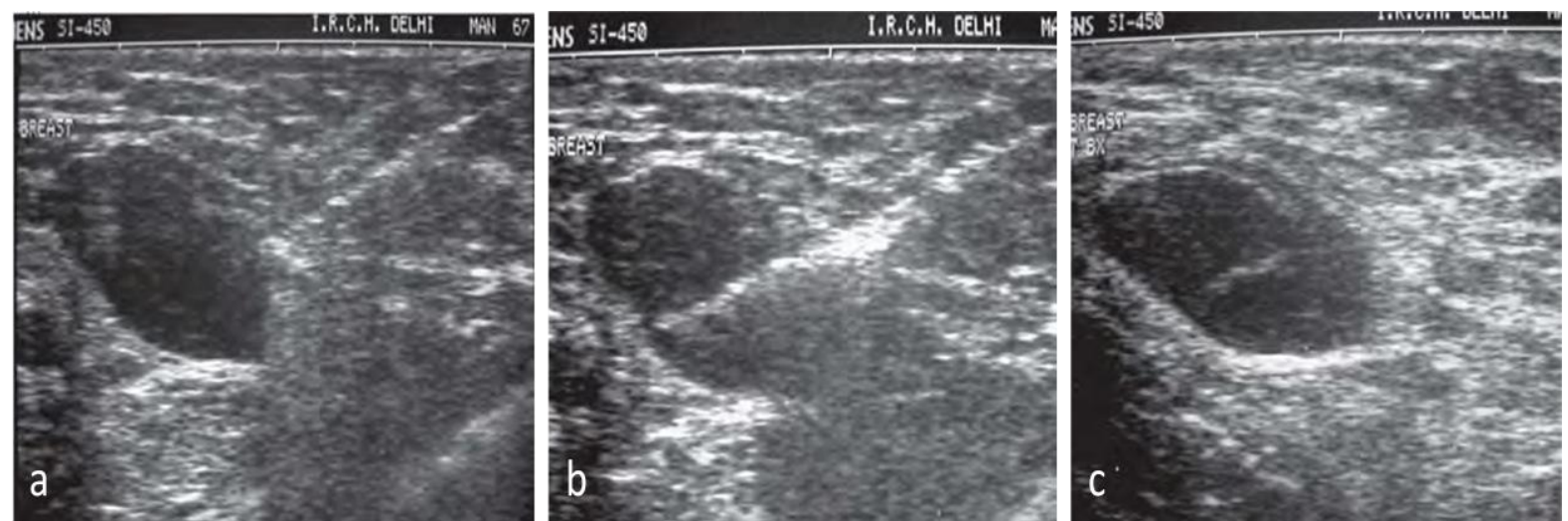

Fig. 6a to c: Ultrasound guided CNB. (a) The tip of the biopsy needle is positioned at the periphery of the mass (b) The mass and the needle are stabilised and the needle is fired. Area of sample notch is seen within the centre of the mass indicating accurate positioning and sampling (c) after withdrawal of the needle, small echogenic area of haemorrhage is seen within the mass at the site of biopsy
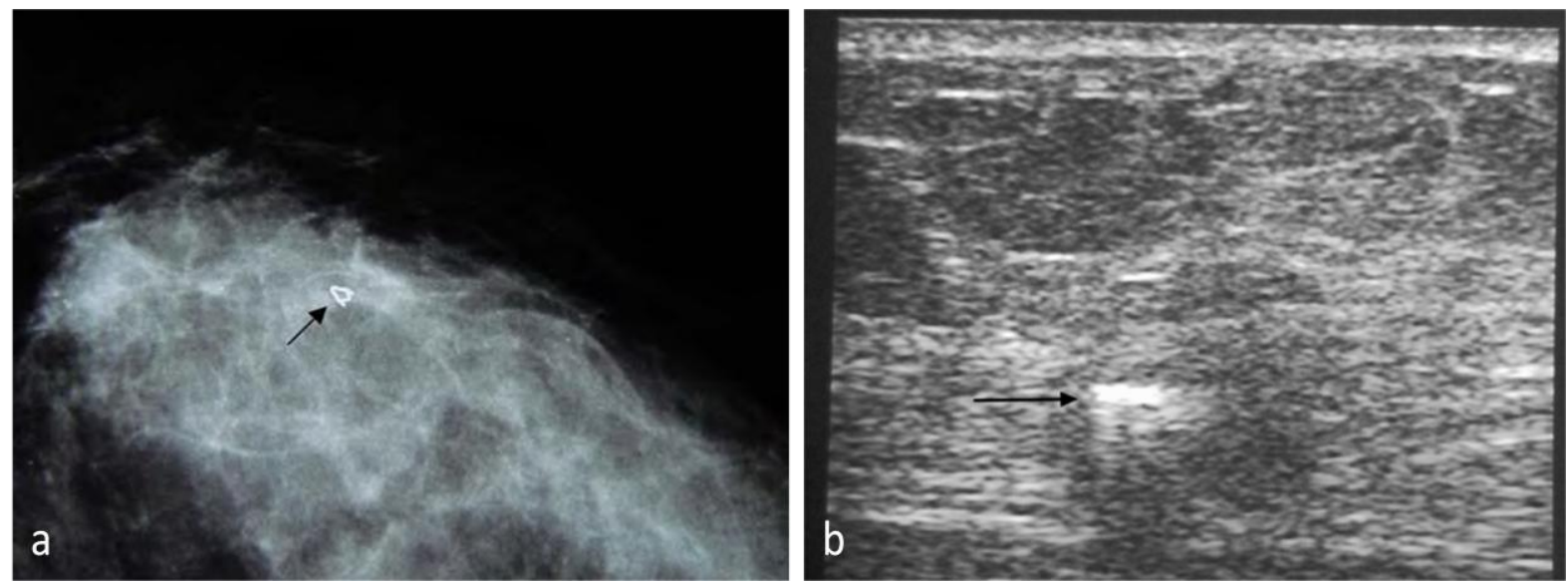

Fig. 7 a \& b: Marker Clips: (a) metallic marker clip Mammomark ${ }^{\circledR}$ deployed at the biopsy site (arrow) after Vacuum Assisted Biopsy is seen on post biopsy mammogram. (b) Gel coated marker clip (arrow) can also be visualized on ultrasound.

be deployed through the biopsy needle when it is likely that the entire lesion has been removed (Fig. 7). If biopsy result is positive, this clip can be targeted for preoperative hook wire localization and surgical excision. The biopsy results must be correlated with imaging features by the radiologist. All biopsy results which are discordant with imaging and/or clinical features need repeat biopsy. ${ }^{25}$ A discordant result may represent sampling error. If the percutaneous biopsy result for a strongly suspicious mammographic lesion (BI-RADS 5) is benign, a repeat biopsy, preferably an excisional biopsy should be undertaken to avoid missing of the cancer. Majority of the lesions with intermediate concern for malignancy (BI-RADS 4a, 4b) are actually benign and hence, benign biopsy result is generally reliable in these. However, regular imaging follow up of these lesions is necessary to ensure that a cancer is not missed. The biopsy result reported as atypical ductal hyperplasia should also be excised as many of these lesions are then upgraded to in-situ or invasive breast cancer. 

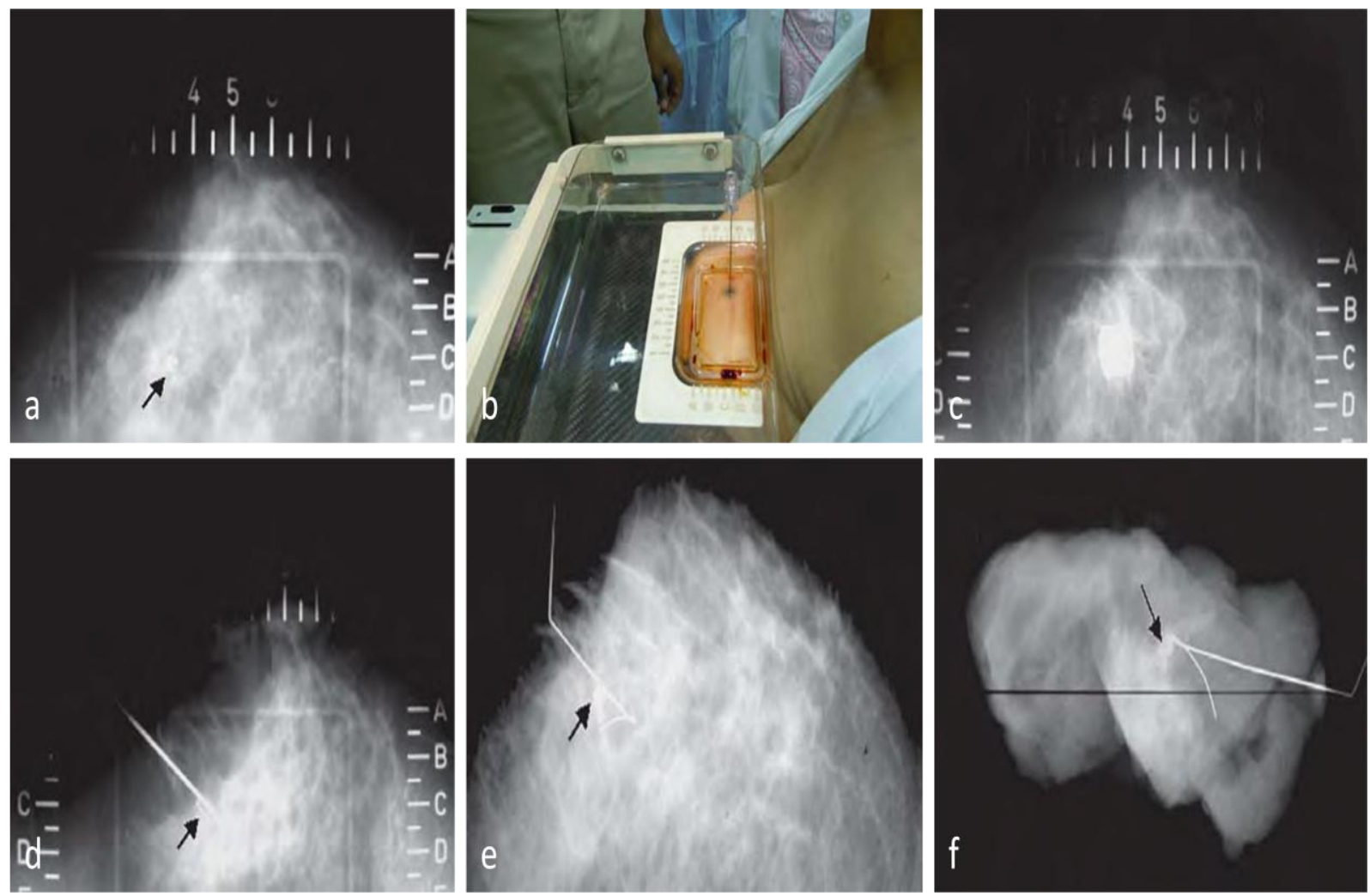

Fig. 8 a to f: pre-operative hook wire localization: (a) Cranio-caudal scout view shows cluster of calcification (arrow) in the centre of the window of the compression plate. Its coordinates are determined in this film and skin entry point is marked. (b) Needle is inserted vertically. Note that the needle entry point is overlapped by the shadow of the hub; ensuring correct needle alignment in the direction of x-ray beam. (c) Another film is obtained. Needle is seen end-on with its hub overlapping the lesion in this view. (d) Medio-lateral view obtained thereafter shows that the needle tip has just crossed the lesion (arrow). Then the hook-wire is inserted and the needle is withdrawn. (e) Post hook wire localization mammogram shows hook wire anchored correctly within the lesion (arrow). (f) Specimen radiograph of the excised tissue confirms accurate removal of the lesion (arrow).

Preoperative Hook wire localization

Preoperative hookwire localization is a procedure in which metallic hook wire is anchored in the non palpable breast lesion so that it can be accurately excised. The excision may be diagnostic i.e. for surgical excisional biopsy or therapeutic i.e. to provide intra-operative guidance to the surgeon during wide local excision for BCT. This would enable accurate removal of the malignant lesion with minimal removal of surrounding normal breast tissue and hence, better cosmesis.

The procedure is traditionally done under mammographic guidance (Fig. 8), but it can also be done under ultrasound or MRI guidance, visibility of the lesion will decide the section of the guiding modality. The post procedure film as well as in-situ hook wire guides the surgeon to reach at the lesion and remove it accurately. The excised specimen is normally subjected to specimen 
Table 1: Breast Imaging Reporting and Database System (BIRADS) Categories.

\begin{tabular}{|c|c|c|}
\hline Category & Assessment & Follow-up Recommendation \\
\hline \multicolumn{3}{|c|}{$\begin{array}{l}\text { a) Assessment is } \\
\text { incomplete }\end{array}$} \\
\hline $\mathbf{0}$ & Assessment is Incomplete & $\begin{array}{l}\text { Need Additional Imaging } \\
\text { Evaluation and/or Prior } \\
\text { Mammograms for Comparison }\end{array}$ \\
\hline \multicolumn{3}{|c|}{$\begin{array}{l}\text { b) Assessment } \\
\text { is Complete - } \\
\text { Final } \\
\text { Categories }\end{array}$} \\
\hline 1 & Negative & $\begin{array}{l}\text { Routine annual screening } \\
\text { mammography (for women } \\
\text { over age } 40 \text { ) }\end{array}$ \\
\hline 2 & Benign Finding(s) & $\begin{array}{l}\text { Routine annual screening } \\
\text { mammography (for women } \\
\text { over age } 40 \text { ) }\end{array}$ \\
\hline 3 & Probably Benign Finding & $\begin{array}{l}\text { Initial short-term follow up } \\
\text { (usually 6-month) Examination }\end{array}$ \\
\hline 4 & $\begin{array}{l}\text { Suspicious Abnormality - Biopsy } \\
\text { Should Be Considered } \\
\text { Optional subdivisions: } \\
\text { 4A: Finding needing intervention with } \\
\text { a low suspicion for malignancy } \\
\text { 4B: Lesions with an intermediate } \\
\text { suspicion of malignancy } \\
\text { 4C: Findings of moderate concern, but } \\
\text { not classic for malignancy }\end{array}$ & Usually requires biopsy \\
\hline 5 & $\begin{array}{l}\text { Highly Suggestive of Malignancy - } \\
\text { Appropriate Action Should Be Taken }\end{array}$ & $\begin{array}{l}\text { Requires biopsy or surgical } \\
\text { treatment }\end{array}$ \\
\hline 6 & $\begin{array}{l}\text { Known Biopsy-Proven Malignancy - } \\
\text { Appropriate Action Should Be Taken }\end{array}$ & $\begin{array}{l}\text { Category reserved for lesions } \\
\text { identified on imaging study with } \\
\text { biopsy proof of malignancy prior } \\
\text { to definitive therapy }\end{array}$ \\
\hline
\end{tabular}

mammography, to confirm the complete removal of the lesion in excised specimen.

\section{Preoperative evaluation of the breast}

Breast cancer is staged according TNM staging system of AJCC. After the diagnosis of breast cancer, clinical staging is performed based on both clinical as well as imaging findings. The clinical stage is denoted as cTNM. For metastatic work-up, chest $\mathrm{x}$-ray, ultrasound of the abdomen and bone scan is sufficient in stage I and II tumours. CT or PET is recommended for stage III cancers.

Surgery, with appropriate adjuvant therapy using hormones, chemotherapy and radiotherapy is the standard treatment of breast cancer. Patients with locally advanced breast cancer are easily diagnosed with clinical breast examination and FNAC, without mandatory contribution of imaging for the diagnosis. However, pre-treatment imaging and interventions, in the form of 
good core biopsy for assessment of tumour grade and ER/PR status is required as this information may not be available on postoperative histopathological examination. A clip placement before initiation of NACT is also required.

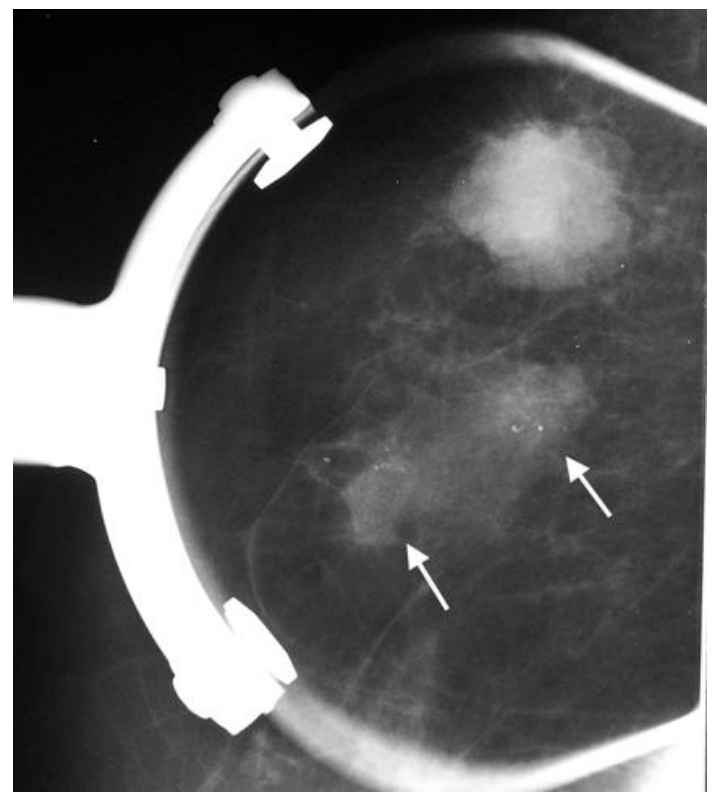

Fig. 9: Multifocal breast cancer: Spot compression mammogram shows two small ill defined masses with microcalcifications (arrows) adjacent to an already diagnosed microlobulated index tumour.

Mastectomy is fast being replaced with breast conserving treatment, BCT (wide local excision with axillary nodal clearance followed by radiotherapy) in suitable women because of its cosmetic benefits and survival advantage which is comparable to mastectomy. All patients diagnosed with breast cancer must be subjected to preoperative breast imaging to select $\mathrm{BCT}$ versus mastectomy.

Main purpose of the mammographic evaluation prior to $\mathrm{BCS}$ is to assess the extent and size of the tumour and to exclude multiple cancers (Fig. 9). Breast cancers that are larger than $5 \mathrm{~cm}$ are generally not treated with BCT. Mammography detects additional cancer in about $10 \%$ patients. Multifocal (located in same quadrant or within $4 \mathrm{~cm}$ ) tumour can be considered for BCT. However, the extent of surgical resection will be wide and may not achieve the desired cosmesis. Also the women with multiple cancers have propensity to develop more cancers after the surgery and hence may fail the treatment. Multicentric (located in different quadrant) cancer is an absolute contraindication for BCT. Presence of suspicious calcification in and around the tumour should be carefully assessed. Clustered calcifications away from the tumour may indicate skip tumour areas. Invasive carcinoma frequently has associated component of DCIS which might extend along the ducts over large area away from the tumour. This is known as extensive intraductal component (EIC). It is a frequent cause of positive margins in surgically resected specimen. Similar to the tumor size, tumor grade and nodal status, EIC is also considered as an important determinant of the prognosis in breast cancer. EIC is diagnosed on postoperative histopathology. Presence of linear branching calcification extending from the primary tumor is a strong indicator of presence of EIC and hence this information is useful while planning the extent of surgery in BCT. Contralateral breast must also be carefully evaluated on mammography to detect additional cancer, if any.

Preoperative ultrasound evaluation of the axilla should be performed for all patients being investigated for early invasive breast cancer and, if morphologically abnormal 
lymph nodes are identified, ultrasoundguided needle sampling should be offered.

Breast MRI is superior to mammography for preoperative evaluation of the both breasts (Fig. 10). Routine preoperative MRI in women already diagnosed with breast cancer detects mammographically occult ipsilateral multicentric cancer in 20\% women and contralateral synchronous cancer in 3-5\% women. ${ }^{26}$ Extent of surgical resection is may be increased if multifocal cancer is detected. Similarly, BCS is converted into mastectomy if multicentric cancer is detected on MRI. Women in whom contralateral breast cancer is detected by MRI are also benefitted as both cancers can be treated at the same time. Overall, preoperative breast MRI changes surgical management in $13 \%$ women, however, it may be due to false positive MRI findings in half of these. False positive MRI, which results in unnecessarily more extensive breast surgery or mastectomy, is an important area of concern. MRI is also useful for assessment of pectoralis muscle in patients with deep seated tumours, as this cannot be evaluated on mammography. Abnormal enhancement of the muscle is a specific indicator of muscle invasion, loss of fat plane is unreliable to diagnose muscle invasion.

The routine use of MRI of the breast is not recommended in the preoperative assessment of patients with biopsy-proven invasive breast cancer or DCIS. MRI of the breast should be offered to patients with invasive breast cancer:

- if there is discrepancy regarding the extent of disease from clinical examination, mammography and ultrasound assessment for planning treatment

- if breast density precludes accurate mammographic assessment

- to assess the tumour size if breast conserving surgery is being considered for invasive lobular cancer ${ }^{27}$

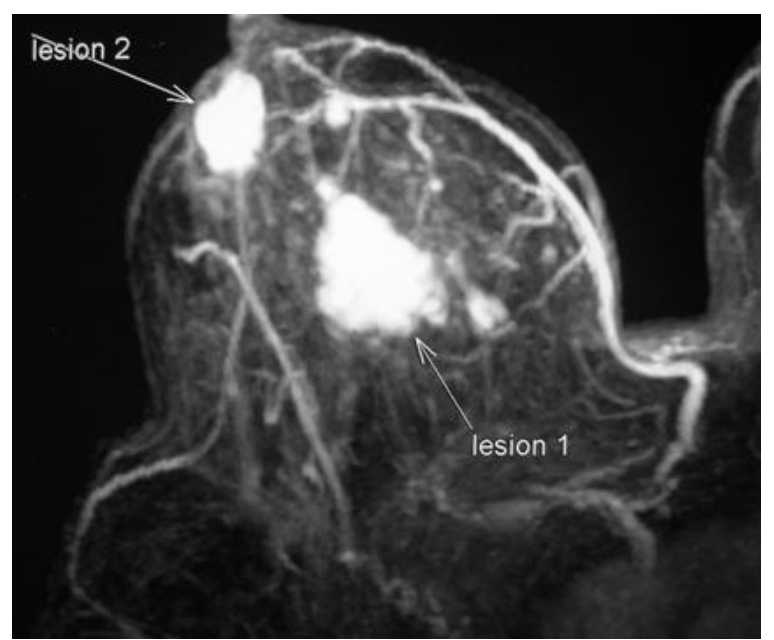

Fig. 10: Mutlicentric breast cancer: MIP image of a contrast enhanced MRI shows a subareolar cancer in addition to an already diagnosed index tumour.

\section{Neoadjuvant chemotherapy}

Breast cancers without distant metastases but with large tumors fixed to skin and/or chest wall, matted fixed axillary lymph nodes and inflammatory breast cancers are traditionally considered as locally advanced breast cancer (LABC) (Fig. 11). Many patients with large $(>5 \mathrm{~cm})$ tumors are resectable but surgery may extensive and difficult in these with high chances of positive margins. These tumors are also included in LABC. Suitable patients with LABC are treated with neoadjuvant chemotherapy with intent of downstaging the tumor. If successful, these patients are then considered for mastectomy or BCT depending on the size of the residual tumor. After completion of the NACT, residual tumour size is an important 
consideration for surgical planning. For this purpose, combination of mammography and ultrasound is more useful than the clinical assessment. MRI is most accurate. It is prudent to insert a metallic marker clip in these tumors before starting the neoadjuvant chemotherapy, preferably done during the biopsy. Many of these patients achieve radiological complete response i.e. the tumor may not be visualized on mammography. In such situations, the metallic marker clip is then used to perform preoperative hook wire localization and local excision.

\section{Peri/Intra operative assessment}

Breast imaging and interventional procedures may also be required in peri/intraoperative phase. Preoperative wire localization, intraoperative ultrasound have important and established role in select women with breast cancer. A close and continuous interaction with surgeon for these procedures improves the accuracy and outcome of BCS.

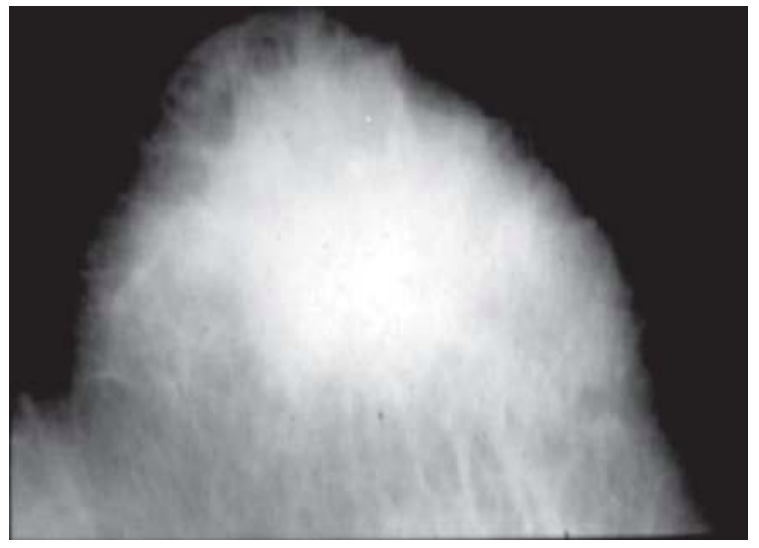

Fig. 11: Inflammatory breast cancer: Mammogram shows diffuse increase in breast density along with thickening of trabeculi and skin. No definite mass is seen.

Non palpable breast cancers undergo breast conserving surgery with prior hook wire localization Palpable tumors do not require this procedure. Specimen mammography is always performed if the breast cancer has calcifications. The specimen mammogram is performed while the patient is still on operating table and is compared with preoperative mammogram to ensure that the abnormalities are completely included in the excised specimen. If the calcifications or mass extend to the margin of the specimen, further resection is required. Specimen mammography is not useful if the cancer was demonstrated as an asymmetry or architectural distortion only on the preoperative mammogram. Although uncommonly utilized, intra-operative ultrasound is useful to assess gross tumour margins more accurately. Use of intraoperative ultrasound results in better margins of resection and lowers the chances of residual tumour.

Post operative but pre radiation mammograms are valuable for post operative assessment. These may be difficult to perform and interpret in a recently operated breast. The breast is tender and adequate compression cannot be given to recently operated breast. The post operative hematoma and edema also makes assessment of the breast difficult and residual mass, if any, cannot be reliably excluded on mammogram. Diagnosis of residual breast cancer after surgery and the decision to reexcise is primarily based on detection of positive tumour margins on post-operative histopathology finding and not on imaging. However, post-operative MRI after positive margins is desirable, if it was not performed pre-operatively. It can identify gross residual tumour or unsuspected multifocal cancer and aid in planning of the extent of re-excision or to consider mastectomy. 


\section{Post Treatment Breast Imaging and Follow Up}

Regular imaging follow-up is required in women treated for breast cancer. Mammography is the standard investigation to detect ipsilateral recurrence or metachronous contra lateral cancer. The baseline mammogram of the treated breast is obtained at 3 to 6 months after completion of the radiotherapy. In case of tender, edematous breast it can be delayed further. This mammogram is compared with the future mammograms. ${ }^{28}$

Several post surgery and radiotherapy changes are apparent on follow up mammogram after BCT. The fibrous scar develops at the operative site which is seen as ill defined density with adjacent architectural distortion (Fig. 12). It may have spiculated appearance and appear suspicious. Exact site of the lumpectomy site must be known and review of preoperative mammograms is helpful for proper evaluation. Careful evaluation in both $\mathrm{CC}$ and MLO views is also helpful. Unlike solid mass, the scar is a two dimensional structure formed by approximation and fibrosis of the lumpectomy cavity. Hence, size and density of the scar varies considerably in two views. Seroma is fluid collection at operative site. It is usually seen as round or oval soft tissue density mass on mammography which may persist for up to two years and the diagnosis is easily established by ultrasound (Fig. 13).

Radiation changes are seen in the form of diffuse thickening of the trabeculi and the skin along with coarseness of the breast parenchyma and an overall increase in the breast density. These changes are similar to the breast edema due any other cause. The severity of these changes is variable in different patients however; these remain stable or decrease on follow up mammograms. Some distortion of the breast outline is also apparent after BCT and the degree of these depends on the extent of the excision. In addition to above changes, there may be development of calcifications in post treatment mammograms. The causes include fat necrosis, sutural calcifications, radiation induced or dystrophic calcifications.

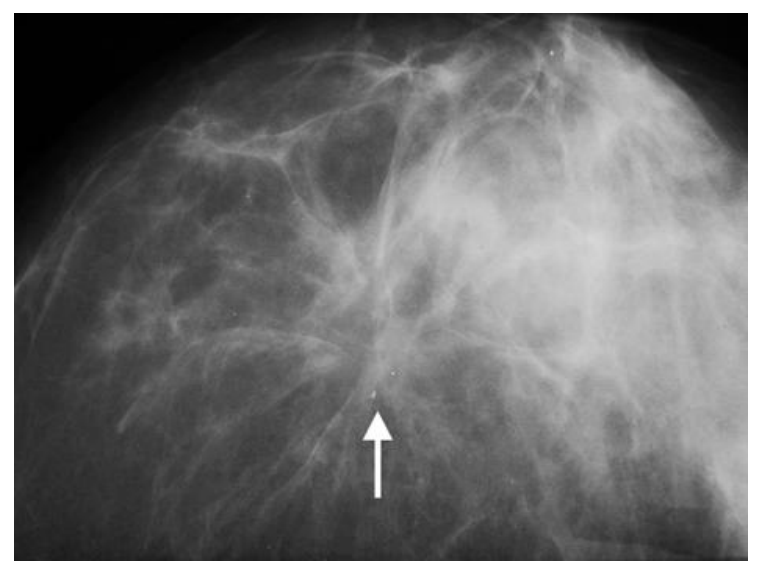

Fig. 12: Postoperative scar: Mammogram shows a spiculated scar (arrow) at the operative site. This is an expected finding and does not suggest a recurrence

Metallic surgical clips may also be found at the margin of the resection. These are intended to mark the lumpectomy area to which boost dose of radiation is given. Local recurrences occur at the rate of $1 \%$ to $2 \%$ per year. Although mammography is the primary modality of follow up and detection of recurrences; its accuracy is lower because of distortion by the post treatment changes. Majority of local recurrences are detected by mammography or by patients themselves on self breast examination, while few are diagnosed on clinical breast examination. The recurrences are most often seen as increased density and/or size of the scar, new mass or suspicious calcifications on 

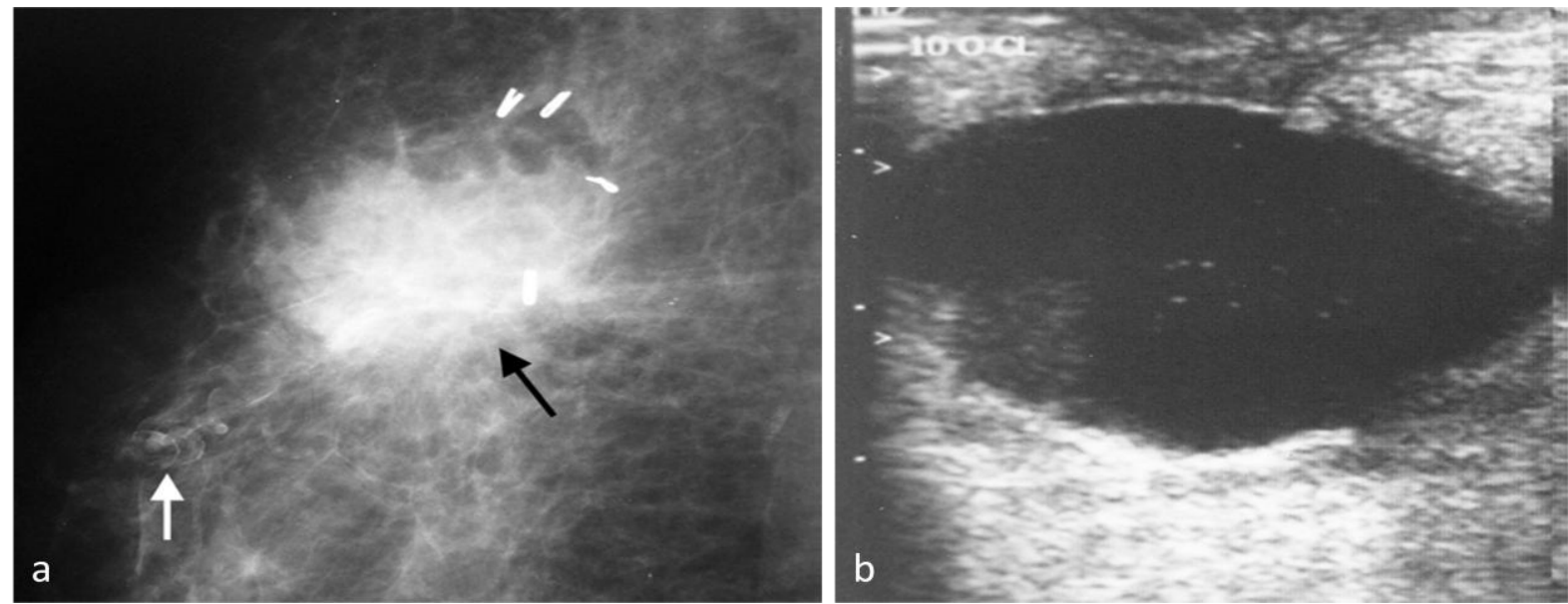

Fig. 13 a \& b: Seroma: (a) Mammogram shows an irregular mass at operative site (black arrow). Adjacent sutural calcification is also seen (white arrow). (b) Ultrasound shows it as a cyst with internal debris.

mammography. Their morphology is usually similar to that of the primary breast cancer in these patients and hence, review of the preoperative mammogram is useful for detection. MRI and percutaneous biopsy should be promptly obtained in case of suspicion of recurrence on mammograms.

Patients treated with mastectomy are also followed with annual screening mammograms of the contralateral breast, as they are at high risk for development of second contralateral breast cancer. Breast cancer survivors have three fold increased risk of developing contralateral breast cancer.

\section{Summary and conclusion}

Diagnostic breast imaging is an integral part of optimal management of breast cancer and other diseases of the breast. A reasonably efficient breast imaging practice can be developed using mammography and ultrasound at any less resource setup if a systematic, quality conscious and protocol based approach is followed. With growing expertise and volumes, the ultimate aim may be the screening for breast cancer in some form, in order to provide full benefits of breast imaging to deserving women.

\section{References}

1. Mushlin AI, Kouides RW, Shapiro DE. Estimating the accuracy of screening mammography: a meta-analysis. Am J Prev Med 1998;14:143-53.

2. Boyd NF, Guo H, Martin LJ. Mammographic density and the risk and detection of breast cancer. N Engl J Med 2007;356:227-36.

3. Tabár L, Vitak B, Chen TH. Swedish two-county trial: impact of mammographic screening on breast cancer mortality during 3 decades. Radiology 2011;260(3):658-63.

4. Otto SJ, Fracheboud J, Verbeek AL, Boer R, Reijerink-Verheij JC, Otten JD, et al. National Evaluation Team for Breast Cancer Screening. Mammography screening and risk of breast cancer death: a population-based case-control study. 
Cancer Epidemiol Biomarkers Prev. 2012 Jan;21(1):66-73.

5. Hari S, Bhalla AS, Thulkar S. Benign and Malignant lesions of the breast. In: Chowdhury V, Gupta AK, Khandelwal N (eds). Diagnostic Radiology: Musculoskeletal \& Breast Imaging. 3rd edition. Delhi: Jaypee Publishers, 2012:482-507.

6. American College of Radiology. ACR BI-RADS: mammography. In: ACR Breast Imaging Reporting and Data System, Breast Imaging Atlas. 4th ed. Reston, Va: American College of Radiology, 2003.

7. Jan M, Mattoo JA, Salroo AN, Ahangar S. Triple assessment in diagnosis of breast cancer in Kashmir. Indian J Surg 2010;72:97-103.

8. Ghimire B, Khan MI, Bibhusal T, Singh Y, Sayami P. Accuracy of triple test score in the diagnosis of palpable breast lump. JNMA J Nepal Med Assoc 2008; 47(172):189-192.

9. McDonald S, Saslow D, Alciati MH. Performance and reporting of clinical breast examination: a review of the literature. CA Cancer J Clin. 2004;54(6):345-361.

10. American College of Radiology. ACRAppropriatenessCriteria:palpablebre astmasses.http://www.acr.org/Secondary MainMenuCategories/quality_safety/app _criteria/pdf/ExpertPanelonWomensIma gingBreastWorkGroup/PalpableBreastM assesDoc3.aspx. Accessed October 25, 2013.
11. Moss HA, Britton PD, Flower CD, Freeman $\mathrm{AH}$, Lomas DJ, Warren RM. How reliable is modern breast imaging in differentiating benign from malignant lesions in symptomatic population? Clin Radiol 1999;54(10):676-682.

12. Salzman B, Fleegle S, Tully AS. Common breast problems. American Family Physician 2012;86(4):343-349.

13. Srivastava A, Mansel RE, Arvind N, Prasad K, Dhar A, Chabra A. Evi $\neg$ dencebased management of mastalgia: a metaanalysis of randomised trials. Breast. 2007;16(5):503-512.

14. Tejwani P, Srivastava A, Nerkar H, Dhar A, Hari S, Thulkar S, et al. Centchroman regresses mastalgia: a randomized comparison with Danazol. Indian J Surg 2011;73(3):199-205.

15. Seo HRN, Na KY, Yim HE, Kim TH, Kang DK. Differential diagnosis in idiopathic granulomatous mastitis and tubercular mastitis. J Breast Cancer 2012;15(1):111-118.

16. Kalu ON, Chow C, Wheeler A, Kong C, Wapnir J. The diagnostic value of nipple discharge cytology: breast imaging compliments predictive value of nipple discharge cytology. J Surg Oncol 2012; 106(4):381-385.

17. Ferris-James DM, Iuanow E, Mehta TS, Shaheen RM, Slanetz PJ. Imaging approaches to diagnosis and management of common ductal abnormalities. Radiographics. 2012 Jul;32(4):1009-30. 
18. Sabel MS, Helvie MA, Breslin T, Curry A, Diehl KM. Is duct excision still necessary for all cases of suspicious nipple discharge? Breast J 2012;18(2):157-162.

19. Guhan-Bilgen I, Okay A. Paget's disease of the breast. Clinical, mammographic, sonographic and pathologic findings in 52 cases. Eur J Radiol 2006;60:256-263.

20. Nicholson BT, Harvey JA, Cohen MA. Nipple-areolar complex: Normal anatomy and benign and malignant processes. Radiographics 2009;29:509523.

21. Olson JA Jr, Morris EA, Van Zee KA. Magnetic resonance imaging facilitates breast conservation for occult breast cancer. Ann Surg Oncol 2000;7:411-15.

22. Youk JH, Kim EK, Ko KH, Kim MJ. Asymmetric mammographic findings based on the fourth edition of BI-RADS: types, evaluation, and management. Radiographics. 2009 Jan-Feb;29(1):e33.

23. American College of Radiology. ACR BI-RADS: mammography. In: ACR Breast Imaging Reporting and Data System, Breast Imaging Atlas. 4th ed. Reston, Va: American College of Radiology, 2003.
24. Tse MG, Tan PH. Diagnosing breast lesions by fine needle aspiration cytology or core biopsy: which is better? Breast cancer Res Treat 2010;123:1-8.

25. Joulaee A, Kalantari M, Kadivar M. Trucut biopsy of breast lesions: the first step toward international standards in developing countries. Eur J Cancer. 2012 Mar; 48(5):648-54.

26. Bilimoria KY, Cambic A, Hansen NM, Bethke KP. Evaluating the impact of preoperative breast magnetic resonance imaging on the surgical management of newly diagnosed breast cancers. Arch Surg 2007;142:441-445.

27. Sardanelli F, Boetes C, Borisch B, Decker T, Federico M. Magnetic resonance imaging of the breast: recommendations from theEUSOMA working group.Eur J Cancer. 2010 May;46(8):1296-316.

28. Thulkar S, Hari S. Present role of mammography/ digital mammography in breast cancer management. PET Clinics 2009;4:213-25. 